\title{
On open maps of Borel sets
}

\author{
by
}

\author{
A. V. Ostrovsky (St. Petersburg)
}

\begin{abstract}
We answer in the affirmative [Th. 3 or Corollary 1] the question of L. V. Keldysh [5, p. 648]: can every Borel set $X$ lying in the space of irrational numbers $\mathbb{P}$ not $G_{\delta} \cdot F_{\sigma}$ and of the second category in itself be mapped onto an arbitrary analytic set $Y \subset \mathbb{P}$ of the second category in itself by an open map? Note that under a space of the second category in itself Keldysh understood a Baire space. The answer to the question as stated is negative if $X$ is Baire but $Y$ is not Baire.
\end{abstract}

Introduction. In 1934 Hausdorff proved [3; 2, 4.5.14] that if $f: X \rightarrow Y$ is an open map from a completely metrizable space $X$ onto a metrizable $Y$, then $Y$ is also completely metrizable. Thus, open maps preserve the class $G_{\delta}$ of Borel sets. L. V. Keldysh proved [5, Th. 1] that this result is not true for Borel sets of higher class, namely, that there is a Borel set $X \subset \mathbb{P}$ of the first category for which there is an open map $f: X \rightarrow Y$ onto an arbitrary analytic set $Y \subset \mathbb{P}$ (see Theorem 1). In connection with this result a question was raised whether an analogous theorem holds for Baire spaces.

It is clear that if $f: X \rightarrow Y$ is an open map and $O \subset Y$ is an open (nonempty) set of the first category, so is $f^{-1}(O)$. Hence, open maps preserve the property of being a Baire space. Let $X_{0} \subset \mathbb{P}$ be an analytic set such that $\mathbb{P} \backslash X_{0}$ does not contain a copy of the Cantor set $\mathbb{C}$. It is not hard to see that $X_{0}$ is a Baire space. Keldysh remarked that if $Y$ satisfies the following condition:

(i) $Y \subset \mathbb{P}$ is an analytic set such that $M \backslash Y$ contains a copy of the Cantor set $\mathbb{C}$ for every $G_{\delta}$-set $M \supset Y$,

then $X_{0}$ cannot be mapped onto $Y$ by an open map [5]. Note that every Borel (non- $G_{\delta}$ ) set $Y \subset \mathbb{P}$ (and analytic set $Y=X_{0} \times \mathbb{P}$ in which every $G_{\delta}$-subspace is Baire [12, Theorem 4]) satisfies the condition (i).

1991 Mathematics Subject Classification: 54H05, 54C10, 54C20, 03E15.

Key words and phrases: open maps, Borel sets, analytic sets, space of the first category, space of the second category, Baire space. 
All spaces in this paper are assumed to be metrizable, and all maps are continuous and onto. We denote by $\mathbb{P}$ and $\mathbb{Q}$ the spaces of irrational and rational numbers, respectively, and by $B(\tau)$ the Baire space of weight $\tau$ (= the Cartesian product of countably many discrete spaces of cardinality $\left.\tau \geqslant \aleph_{0}\right)$. It is known that every metrizable space $X$ with Ind $X=0$ and $w(X)=\tau \geqslant \aleph_{0}$ can be embedded in $B(\tau)$ (for $\tau=\aleph_{0}, B(\tau)=\mathbb{P}$ ) [2, Theorem 7.3.15].

A set $Y \subset \mathbb{P}$ is called an analytic set (respectively, a Borel set) if there exists a map $f: \mathbb{P} \rightarrow Y$ (respectively, a one-to-one map $f: M \rightarrow Y$, where $M$ is a $G_{\delta}$-set in $\left.\mathbb{P}\right)$.

The notation $X \hookleftarrow Y$ means that $X$ contains a relatively closed subset which is homeomorphic to $Y$, the symbol $\approx$ denotes a homeomorphism, and $[A]$ denotes the closure of $A$.

The space $X$ is called of the first category (respectively, of the second category) if $X$ can (respectively, cannot) be represented as a countable union of nowhere dense (n.d.) sets in $X$.

We say that $X$ has a property $L$ everywhere if every open subspace $U \subset X$ has property $L$. The space $X$ is Baire iff $X$ is everywhere of the second category. A subset of $X$ is clopen if it is both closed and open in $X$.

We say that a pair of spaces $X, Y$ is exceptional if either

(a) $X$ is Baire and $Y$ is not, or

(b) $Y$ is of the first category and $X$ is not.

It is clear that if there is an open map $f: X \rightarrow Y$ then $X, Y$ is not an exceptional pair.

The following theorem gives a necessary and sufficient condition on Borel sets $X, Y \subset \mathbb{P}$ for the existence of an open map $g: X \rightarrow Y$; it shows that the answer to the Keldysh question [5] is affirmative.

Theorem 0. Let $X \subset \mathbb{C}$ be a Borel set, $Y \subset \mathbb{C}$ be an analytic set, and $X$ be everywhere not $F_{\sigma} \cup G_{\delta}$. Then there exists an open map $f: X \rightarrow Y$ if and only if $X, Y$ is not an exceptional pair.

It is not hard to see that Theorem 0 is the sum of Theorems $1-4$, and Saint Raymond's theorem [14, Theorem 5]: Let $X$ be a Borel set in $\mathbb{C}$; then $X$ is a union of $F_{\sigma}$ and $G_{\delta}$ (in $\mathbb{C}$ ) iff $X \nleftarrow \mathbb{P} \times \mathbb{Q}$. It can be seen that Theorem 4 is based on Theorems 1 and 2, and Theorem 3 uses Theorem 2, which uses Theorem 1. Lemma 2 and the first step of its proof strengthen the theorem of [10].

Re mark. If $X, Y \subset \mathbb{C}, X$ contains an open $F_{\sigma} \cup G_{\delta}$ (relative to $\mathbb{C}$ ) and $f: X \rightarrow Y$ is an open map then $Y$ also contains an open $F_{\sigma} \cup G_{\delta}$ (relative to $\mathbb{C})$. 
Indeed, suppose $X$ contains an open $U=X_{1} \cup X_{2}$, where $X_{1}$ is $F_{\sigma}$ and $X_{2}$ is $G_{\delta}$. It is clear that $f\left(X_{1}\right)$ is $F_{\sigma}$ in $\mathbb{C}, T=U \backslash f^{-1}\left(f\left(X_{1}\right)\right)$ is $G_{\delta}$ and $f \nmid T$ is an open map. Hence by Hausdorff's theorem $f(T)$ is $G_{\delta}$ in $\mathbb{C}$ and $f(U)=f(T) \cup f\left(X_{1}\right)$ is $F_{\sigma} \cup G_{\delta}$.

Note that $\mathbb{C}$ is embeddable in $\mathbb{P}$ and if $X \subset \mathbb{C} \subset \mathbb{P}$ is not $F_{\sigma} \cap G_{\delta}$ in $\mathbb{P}$, then $X$ is not $F_{\sigma} \cap G_{\delta}$ in $\mathbb{C}$.

We close this section with an example of a Baire space $X \subset \mathbb{C}$ which is $F_{\sigma} \cup G_{\delta}$ and everywhere not $F_{\sigma} \cap G_{\delta}$. Thus, if $Y$ is any Baire space which is everywhere not $F_{\sigma} \cup G_{\delta}$ then $X, Y$ is not an exceptional pair, but no open $f: X \rightarrow Y$ exists, showing that the condition on $X$ in Theorem 0 cannot be weakened.

Indeed, let $\mathbb{Q}^{\prime} \approx \mathbb{Q}$ be a dense subset of $\mathbb{C}$ and $\mathbb{P}^{\prime}=\mathbb{C} \backslash \mathbb{Q}^{\prime}$. Let now $X_{1}=\mathbb{Q}^{\prime} \times \mathbb{Q}^{\prime}, X_{2}=\mathbb{P}^{\prime} \times \mathbb{P}^{\prime}$ and $X=X_{1} \cup X_{2}$. Obviously $X$ is a Baire space and $F_{\sigma} \cup G_{\delta}$ in $\mathbb{C}$, and $X$ is everywhere not $F_{\sigma} \cup G_{\delta}$ since every $F_{\sigma} \cap G_{\delta}$ in $\mathbb{C}$ which is everywhere not $F_{\sigma}$ and everywhere not $G_{\delta}$ is homeomorphic to $\mathbb{P} \times \mathbb{Q}$ (see [8], [13]) and we have a contradiction to the Baire Category Theorem. (Notice that $X$ is homeomorphic to the space $T$, which has been characterized by van Douwen [1, Theorem 2.3].)

1. Main theorems. The proofs of Theorems 1-4 use Lemmas 2-5 of Section 3, which use Proposition 0 of Section 2.

Theorem 1. Let $X, Y \subset \mathbb{P}$ be analytic sets, and $X$ be a space of the first category and everywhere not a $\sigma$-compact space. Then there exists an open map $g: X \rightarrow Y$.

Remark. If $X \subset \mathbb{P}$ is an analytic set and $X$ is not a $\sigma$-compact space, then $X \hookleftarrow \mathbb{P}$ and for every analytic set $Y \subset \mathbb{P}$ there exists a map $f: X \rightarrow Y$ $[4 ; 6, \S 39 ; 10$, Corollary 2].

Proof of Theorem 1. According to the above remark, if $U \subset X$ is an open set then $U \hookleftarrow \mathbb{P}$. Since $\mathbb{P} \times \mathbb{P} \approx \mathbb{P}$ we may suppose that $\mathbb{P}$ is n.d. in $X$. Let $X=\bigcup F_{i}^{\prime}$, where $F_{i}^{\prime}$ is closed n.d. in $X(i \in \omega)$. Obviously, for every $F_{i}^{\prime}$ there exists a sequence of closed n.d. (in $X$ ) subsets $P_{i, j} \approx \mathbb{P}$ such that

$$
F_{i}^{\prime}=\left[\bigcup\left\{P_{i, j}: j \in \omega\right\}\right]_{X} \backslash \bigcup\left\{P_{i, j}: j \in \omega\right\},
$$

and every set

$$
F_{i}=F_{i}^{\prime} \cup \bigcup\left\{P_{i, j}: j \in \omega\right\}
$$

is closed n.d. in $X$. The reader can easily verify that every $F_{i}$ is an analytic set and no open $U \subset F_{i}$ is $\sigma$-compact. By the above remark, it remains to apply Lemma 2 . 
Theorem 2. Let $X, Y \subset \mathbb{P}$ be analytic, Baire spaces and everywhere $X \hookleftarrow \mathbb{P} \times \mathbb{Q}$. Then there exists an open map $g: X \rightarrow Y$.

Proof. Every analytic set $X$ can be represented as $X_{1} \cup X_{2}$, where $X_{2}$ is a $G_{\delta^{-}}$set in $\mathbb{P}$ and $X_{1}$ is of the first category in $X[6, \S 11]$. Since $X$ is everywhere of the second category, $\left[X_{2}\right]=X$ and if $U=X \backslash\left[X_{1}\right] \neq \emptyset$ then $U \subset X_{2}$ is a $G_{\delta}$-set in $\mathbb{P}$ and $U \hookleftarrow \mathbb{P} \times \mathbb{Q}$. This implies that $U \hookleftarrow \mathbb{Q}$, which contradicts the Baire Category Theorem. Hence $\left[X_{1}\right]=X$.

Analogously $Y=Y_{1} \cup Y_{2}$, where $Y_{2}$ is a $G_{\delta}$-set in $\mathbb{P}, Y_{1}$ is of the first category, and $\left[Y_{2}\right]=Y$. We may suppose that $\left[Y_{1}\right]=Y$. Indeed, it is clear that there is an open map (projection) $\pi: \mathbb{P} \times Y \rightarrow Y$, hence we may consider $\mathbb{P} \times Y$ instead of $Y$. Taking a dense countable subset $\mathbb{Q}^{\prime} \approx \mathbb{Q}$ in $\mathbb{P} \times Y_{2}$ we set

$$
Y_{1}^{\prime}=\left(\mathbb{P} \times Y_{1}\right) \cup \mathbb{Q}^{\prime} \quad \text { and } \quad Y_{2}^{\prime}=\left(\mathbb{P} \times Y_{2}\right) \backslash \mathbb{Q}^{\prime} .
$$

It is clear that $\mathbb{P} \times Y=Y_{1}^{\prime} \cup Y_{2}^{\prime}$, where $Y_{1}^{\prime}$ is a dense subset of the first category in $\mathbb{P} \times Y$, and $Y_{2}^{\prime}$ is a $G_{\delta^{-}}$set $($in $\mathbb{P} \times \mathbb{P} \approx \mathbb{P}$ ) dense in $\mathbb{P} \times Y$.

Represent $X_{2}$ as

$$
X_{2}=\bigcap\left\{O_{i}: i \in \omega\right\}, \quad \text { with each } O_{i} \text { open in } \mathbb{P},
$$

and let $F_{i}^{\prime}=X \backslash O_{i}$. Similarly to the proof of Theorem 1 (see (A) and (B)) one defines closed n.d. sets

$$
F_{i}=F_{i}^{\prime} \cup \bigcup\left\{P_{i, j}: j \in \omega\right\},
$$

where the $P_{i, j} \approx \mathbb{P} \times \mathbb{Q}$ are closed n.d. sets. Clearly, $F_{i}$ is a subspace of the first category, and for a relatively open set $U \subset F_{i}$ we have $U \hookleftarrow \mathbb{P} \times \mathbb{Q}$. Hence $U$ is not $\sigma$-compact and of the first category. By Theorem 1 there exists an open map of $U$ onto every nonempty analytic set in $Y$. It is clear that $X$ is everywhere not compact and the conditions (i)-(iv) of Lemma 3 hold, hence by Lemma 3 one obtains the assertion.

Lemma 1. Let $X \subset \mathbb{P}$ be a space which is not a Baire space and not of the first category. Then $X=T \cup X_{1}$, where $X_{1}=X \backslash T$ is an open space of the first category, $T=F \cup X_{2}$ is a closed Baire subspace, $F$ is a closed n.d. set in $T$ and in $F \cup X_{1}, X_{2}=T \backslash F$ is an open (in $X$ ) Baire space.

Proof. Define $X_{1}$ as the union of all open subsets of $X$ of the first category. Since $X$ is Lindelöf, $X_{1}$ is a maximal open subspace of the first category. Put $T=X \backslash X_{1}$ and $F=\left[X_{1}\right] \backslash X_{1}$. It is clear that $F$ is n.d. in $\left[X_{1}\right]$, hence in $X$. Obviously, $X_{2}=X \backslash\left[X_{1}\right]=T \backslash F \neq \emptyset$, otherwise $X$ would be of the first category. The subspace $T$ is everywhere of the second category, because there exists no open $V \subset X$ such that $V \cap T \neq \emptyset$ is of the first category in $T$ (otherwise, as $V \cap X_{1}$ is of the first category, $X_{1}$ would not be maximal). Obviously, $X_{2}$ is dense in $T=F \cup X_{2}$, since $\left[X_{1}\right]=X_{1} \cup F$ is of 
the first category in $X$ and if there is an open $V \subset X$ with $\emptyset \neq V \cap T \subset F$, then $V \subset\left[X_{1}\right]$ is of the first category in $X, V \not \subset X_{1}$ and again $X_{1}$ would not be maximal.

Theorem 3. Let $X, Y$ be analytic sets in $\mathbb{P}, Y$ be a Baire space and everywhere $X \hookleftarrow \mathbb{P} \times \mathbb{Q}$. Then there exists an open map $g: X \rightarrow Y$.

Proof. Let $X$ be a space of the first category. Since everywhere $X \hookleftarrow$ $\mathbb{P} \times \mathbb{Q}, X$ is everywhere not $\sigma$-compact by the Baire Category Theorem. Now we apply Theorem 1.

If $X$ is Baire we apply Theorem 2 .

In the third case, by Lemma 1, every open $U \subset X \backslash T$ satisfies the conditions of Theorem 1 and therefore can be mapped onto every open set $V \subset Y$, and $T$ is a closed subspace satisfying the conditions of Theorem 2, hence there exists an open map $f: T \rightarrow Y$. By Lemma 4 , there is an open extension $g: X \rightarrow Y$ of $f$.

Corollary 1. Let $X \subset \mathbb{C}$ be a Borel set everywhere not $F_{\sigma} \cup G_{\delta}$. Then for every analytic Baire space $Y \subset \mathbb{C}$ there exists an open map $g: X \rightarrow Y$.

This follows from Theorem 3, since by the Saint Raymond's Theorem [14, Theorem 5; 7, Corollary 17] for every Borel not $F_{\sigma} \cup G_{\delta}$-set $U \subset X$ we have $U \hookleftarrow \mathbb{P} \times \mathbb{Q}$.

Theorem 4. Suppose that $X, Y$ are analytic sets in $\mathbb{P}$ of the second category and everywhere $X \hookleftarrow \mathbb{P} \times \mathbb{Q}$. Suppose that $X$ and $Y$ contain open (nonempty) subsets of the first category. Then there exists an open map $g: X \rightarrow Y$.

Proof. By Lemma 1, $X=X_{1} \cup F^{X} \cup X_{2}$, where $X_{1}$ is an open subspace of $X$ of the first category, $F^{X}$ is n.d. in $X_{1} \cup F^{X}$ and $X_{2}$ is an open (in $X$ ) Baire subspace such that $F^{X}$ is a n.d. set in $F^{X} \cup X_{2}\left(X_{1} \cap X_{2}=\emptyset\right)$. Analogously we have $Y=Y_{1} \cup F^{Y} \cup Y_{2}$ with the same properties. Similarly to the proofs of Theorems 1 and $2\left(\right.$ see $\left(\mathrm{B}^{\prime}\right)$ and $\left.(\mathrm{B})\right)$ we take a closed n.d. set $F_{0}^{X} \supset F^{X}$ such that there exists an open map $f: F_{0}^{X} \rightarrow F^{Y}$. By Lemma 5 and Theorems 1 and 2, there exist open extensions $g_{1}: F_{0}^{X} \cup X_{1} \rightarrow F^{Y} \cup Y_{1}$ and $g_{2}: F_{0}^{X} \cup X_{2} \rightarrow F^{Y} \cup Y_{2}$ of $f$. Then it is easy to see that $g=g_{1} \cup g_{2}: X \rightarrow Y$ is an open extension of $f$.

\section{Terminology and basic facts}

1.0. We denote by $A^{<\omega}$ the set of all finite sequences $u=\langle u(0), \ldots, u(k)\rangle$ of elements of $A$; $\emptyset$ denotes the empty sequence. The number $|u|=k+1$ is called the length of $u$; define $|\emptyset|=0$.

If $u, v \in A^{<\omega}$, then $u^{\curlyvee} v$ is the concatenation of the two sequences, i.e.

$$
\langle u(0), \ldots, u(k)\rangle^{\curlyvee}\langle v(0), \ldots, v(m)\rangle=\langle u(0), \ldots, u(k), v(0), \ldots, v(m)\rangle .
$$


Of course, $u^{\curvearrowleft} \emptyset=u$. The notation $s \subset t$ means that $t$ extends $s$, i.e. that $s$ is an initial segment of $t$ and $s \neq t$.

A tree $T$ on $A$ is a subset of $A^{<\omega}$ such that $s \in T$ and $t \subset s \rightarrow t \in T$. If $t \subset s$ and $|t|+1=|s|$, we write $s=t^{+}$.

1.1. Let $T \subset B(\tau)$. A $\gamma(T)$-system is a family of open (in $T=T_{\emptyset}$ ) subsets $T_{s}$, indexed by some tree $S$ satisfying the conditions:

(a) $\bigcup\left\{T_{s^{+}}: s^{+} \in S\right\}=T_{s}$;

(b) $\operatorname{diam} T_{s} \rightarrow 0$ as $|s| \rightarrow \infty$.

1.2. A $\gamma^{*}(T)$-system is a $\gamma(T)$-system with the additional condition:

(c) for every fixed $n=1,2, \ldots$ the sets $T_{s},|s|=n$, are pairwise disjoint clopen sets.

Obviously, for every set $T \subset B(\tau)$ there exists a $\gamma^{*}(T)$-system.

2.1. Let now $T \subset X \subset B(\tau)$. A $\delta(T)$-extension of a $\gamma(T)$-system $\left\{T_{s}\right\}$ to $X$ is a family of open sets $X_{s}$ in $X\left(X_{\emptyset}=X\right)$ such that

(d) $X_{s} \cap T=T_{s}$;

(e) $\operatorname{diam} X_{s} \rightarrow 0$ as $|s| \rightarrow \infty$;

(f) the sets

$$
Z_{s}=X_{s} \backslash \bigcup\left\{X_{s^{+}}: s^{+} \in S\right\}
$$

are open in $X$;

$\left(\mathrm{f}_{1}\right) X_{s} \backslash T=\bigcup\left\{Z_{t}: t \supseteq s, t \in S\right\}$;

(g) if $T$ is a nowhere dense subset of $X$ then the sets $Z_{s}$ are nonempty.

2.2. A $\delta^{*}(T)$-extension is a $\delta(T)$-extension with the following additional condition:

(h) the sets $X_{s}$ are pairwise disjoint for every fixed $|s|=n$ and the sets $Z_{s}$ are pairwise disjoint and clopen in $X$.

Proposition 0. Let $T$ be a closed subset of $X \subset B(\tau)$. Then every $\gamma(T)$-system (respectively, $\gamma^{*}(T)$-system) has a $\delta(T)$-extension (respectively, $\delta^{*}(T)$-extension $)$.

Proof. Let $S$ be the tree indexing the given $\gamma(T)$-system.

Fix $s \in S$, and suppose $X_{s}$ has already been constructed (we take $X_{\emptyset}=X$ ). If $T$ is a n.d. set, also take some $x_{0} \in X_{s} \backslash T$. For each $s^{+} \in$ $S$ take an open set $O_{s^{+}} \subset X_{s}$ (with $x_{0} \notin O_{s^{+}}$if $T$ is n.d.) such that $O_{s^{+}} \cap T=T_{s^{+}}$. For every $x \in X_{s} \backslash T$ take a neighbourhood $O(x) \subset X_{s}$ such that $O(x) \cap T=\emptyset$. The cover

$$
\left\{O_{s^{+}}, O(x): x \in X_{s} \backslash T, s^{+} \in S\right\}
$$


of $X_{s}$ has a refinement $\lambda=\left\{U_{\alpha}: \alpha \in A\right\}$, where the $U_{\alpha}$ are clopen in $X$ and pairwise disjoint. Put

$$
Z_{s}=\bigcup\left\{U_{\alpha} \in \lambda: U_{\alpha} \cap T_{s}=\emptyset\right\} .
$$

It is easy to see that

$$
Z_{s}=X_{s} \backslash V_{s}
$$

where

$$
V_{s}=\bigcup\left\{U_{\alpha} \in \lambda: U_{\alpha} \cap T_{s} \neq \emptyset\right\} \subset \bigcup\left\{O_{s^{+}}: s^{+} \in S\right\} .
$$

Define

$$
X_{s^{+}}=O_{s^{+}} \cap V_{s} \text {. }
$$

Then by $(*)$ and $(* *)$,

$$
Z_{s}=X_{s} \backslash \bigcup\left\{O_{s^{+}} \cap V_{s}: s^{+} \in S\right\}=X_{s} \backslash \bigcup\left\{X_{s^{+}}: s^{+} \in S\right\} .
$$

Obviously, we have (d) (for $\left.s^{+}\right)$. We can get condition (e) to be also satisfied, choosing the sets $O_{s}$ in a proper way and taking into account (b).

Conditions (f), $\left(\mathrm{f}_{1}\right)$ hold by the construction, and $(\mathrm{g})$ follows from the fact that $x_{0} \in Z_{s}$.

So, we have proved the existence of the required $\delta(T)$-extension. In the case of a $\gamma^{*}(T)$-system we consider for every $x \in T_{s^{+}}$a neighbourhood $O(x)$ such that $O(x) \cap T \subset T_{s^{+}}$instead of the set $O_{s^{+}}$, and choose $O(x)$ for $x \in X_{s} \backslash T$ and a refinement $\lambda$ as above. Then put

$$
X_{s^{+}}=\bigcup\left\{U_{\alpha} \in \lambda: U_{\alpha} \cap T \subset T_{s^{+}}\right\} .
$$

It is clear that we have (h).

3. Principal lemmas. A map $f: X \rightarrow Y$ is called open at $x \in X$ if there is a base $\mathcal{B}$ for $X$ at $x$ such that $\{f(U): U \in \mathcal{B}\}$ is a base for $Y$ at $f(x)$.

Lemma 2. Suppose $X, Y \subset B(\tau)$ and $X=\bigcup_{i \in \omega} F_{i}$, where the $F_{i}$ are closed nowhere dense sets such that for every nonempty clopen (relative to $\left.F_{i}\right)$ set $V \subset F_{i}(i \in \omega)$ and every nonempty clopen set $U \subset Y$ there exists a map $f: V \rightarrow U$. Then there exists an open map $g: X \rightarrow Y$.

Proof. The proof is by induction. We will define a tree $H$, and for each $h \in H$ closed sets $F_{h} \subset$ some $F_{i}$, clopen subsets $O_{h} \subset X, Y_{h} \subset Y$, maps $g_{h}: F_{h} \rightarrow Y_{h}$, and trees $H_{h}$ such that $h^{\prec}\langle v\rangle \in H$ if and only if $v \in H_{h}$. Always, $h_{n}$ will denote an element of $H$ of length $n$.

At the first step put $F_{\emptyset}=F_{0}, O_{\emptyset}=X, Y_{\emptyset}=Y$ and consider a map $g_{\emptyset}: F_{\emptyset} \rightarrow Y_{\emptyset}$. Suppose that we have already constructed $F_{h_{n}}, O_{h_{n}}, Y_{h_{n}}$, and $g_{h_{n}}$, and $H_{h}$ for all $h \subset h_{n}$. Take a $\gamma^{*}\left(Y_{h_{n}}\right)$-system. It is clear that there 
exist a tree $H_{h}$ and a $\gamma^{*}\left(F_{h_{n}}\right)$-system $\left\{T_{h_{n}} \prec\langle v\rangle: v \in H_{h}\right\}$ such that for each $v \in H_{h}$ there is some $Y_{h_{n}} \prec\langle v\rangle \in \gamma^{*}\left(Y_{h_{n}}\right)$ with $T_{h_{n}} \prec\langle v\rangle \subset g_{h_{n}}^{-1}\left(Y_{h_{n}} \prec\langle v\rangle\right)$. By Proposition 0, let $\left\{X_{h_{n}} \prec\langle v\rangle: v \in H_{h}\right\}$ be a $\delta^{*}\left(F_{h_{n}}\right)$-extension of $\gamma^{*}\left(F_{h_{n}}\right)$ in $O_{h_{n}}=X_{h_{n}} \prec\langle\emptyset\rangle$. Fix $v \in H_{h}$, and put $O_{h_{n}} \prec\langle v\rangle=Z_{h_{n}} \prec\langle v\rangle$, where $Z_{h_{n}} \prec\langle v\rangle$

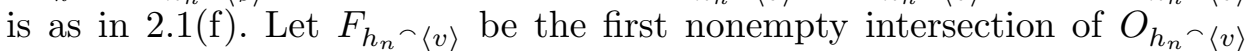
with the sets $F_{i}(i \in \omega)$. Then by our condition there is a map $g_{h_{n}<\langle v\rangle}$ : $F_{h_{n} \frown\langle v\rangle} \rightarrow Y_{h_{n}} \prec\langle v\rangle$. We may put $h_{n} \bumpeq\langle v\rangle=h_{n+1} \in H$ and define the map $g: X \rightarrow Y$ as follows: $g\left\lceil F_{h}=g_{h}\right.$ for all $h \in H$.

FACT. $g\left(X_{h_{n+1}}\right)=g\left(F_{h_{n+1}}\right)=Y_{h_{n+1}}$.

Indeed, by construction

$$
X_{h_{n+1}}=T_{h_{n+1}} \cup \bigcup\left\{F_{p_{n+k+1}}: F_{p_{n+k+1}} \subset X_{h_{n+1}}, p_{n+k+1} \in H, k \in \omega\right\}
$$

and for every $F_{p_{n+k+1}} \subset X_{h_{n+1}}(k>0)$ there is $F_{p_{n+k}}\left(h_{n} \subset p_{n+k} \subset p_{n+k+1}\right)$ such that $g\left(F_{p_{n+k}}\right) \supset g\left(F_{p_{n+k+1}}\right)$, hence

$$
g\left(X_{h_{n+1}}\right)=g\left(T_{h_{n+1}}\right) \cup \bigcup\left\{g\left(F_{p_{n+1}}\right): F_{p_{n+1}} \subset X_{h_{n+1}}\right\} .
$$

It remains to remark that $g\left(T_{h_{n+1}}\right) \subset g\left(F_{h_{n+1}}\right)$ and for every $F_{p_{n+1}} \subset X_{h_{n+1}}$ we have $g\left(F_{p_{n+1}}\right) \subset Y_{h_{n+1}}=g\left(F_{h_{n+1}}\right)$.

Now, if $x \in X$, then $x \in F_{h_{n}}$ for some $F_{h_{n}}$. Let $\mathcal{B}=\left\{X_{h_{n+1}}: x \in X_{h_{n+1}}\right\}$. By our construction, $\mathcal{B}$ is a base at $x$ and by the fact above $g$ is an open map at $x$.

Lemma 3. Let $X, Y \subset B(\tau)$, and let the following conditions for $X$ and analogous conditions for $Y$ hold:

(i) there exist open sets $U_{i}^{X}(i \in \omega)$ in $B(\tau)$ such that the set $G_{\delta}^{X}=$ $\bigcap\left\{U_{i}^{X}: i \in \omega\right\}$ is dense in $X\left(\right.$ and $\left.G_{\delta}^{X} \subset X\right)$;

(ii) the set $F_{\sigma}^{X}=X \backslash G_{\delta}^{X}$ is dense in $X$;

(iii) for all sets $U \subset F_{i}^{X}$ and $V \subset F_{j}^{Y}$ clopen relative to $F_{i}^{X}=X \backslash U_{i}^{X}$ and $F_{j}^{Y}=Y \backslash U_{j}^{Y}$, respectively, there exists an open map $f: U \rightarrow V$ $(i, j \in \omega)$;

(iv) for all clopen sets $O \subset X, W \subset Y$ and each refinement $\lambda(W)$ of $W$ consisting of clopen (in $Y$ ) pairwise disjoint sets, there is a refinement $\lambda(O)$ of $O$ consisting of clopen (in $X)$ pairwise disjoint sets such that the cardinality of $\lambda(O)$ is greater than or equal to the cardinality of $\lambda(W)$.

Then there exists an open map $g: X \rightarrow Y$.

Proof. The proof is by an inductive process similar to that used to establish Lemma 2.

At step 0 consider an open map $f_{0}: F_{0}^{X} \rightarrow F_{0}^{Y}$ (where $F_{0}^{X}=X \backslash U_{0}^{X}$, $F_{0}^{Y}=Y \backslash U_{0}^{Y}$ ) and put $O_{0}^{X}=X, O_{0}^{Y}=Y$. Obviously, we may suppose that $\operatorname{diam} O_{0}^{X}<1$ and $\operatorname{diam} O_{0}^{Y}<1$. 
Suppose we obtained at step $n$ clopen sets $O_{t_{n}}^{X}$ and corresponding sets $O_{t\left(t_{n}\right)}^{Y}$ with the following properties:

(a) $\operatorname{diam} O_{t_{n}}^{X}<1 / 2^{n}$ and $\operatorname{diam} O_{t\left(t_{n}\right)}^{Y}<1 / 2^{n}$;

(b) $\left[O_{t_{n}}^{X}\right] \subset U_{n-1}^{X}$ and $\left[O_{t\left(t_{n}\right)}^{Y}\right] \subset U_{n-1}^{Y}\left(U_{-1}^{X}=U_{-1}^{Y}=B(\tau)\right)$.

We also obtained closed n.d. sets

$$
F_{t_{n}}^{X} \subset O_{t_{n}}^{X}, \quad F_{t\left(t_{n}\right)}^{Y} \subset O_{t\left(t_{n}\right)}^{Y}
$$

and maps

$$
f_{t_{n}}: F_{t_{n}}^{X} \rightarrow F_{t\left(t_{n}\right)}^{Y}
$$

Consider some $\gamma^{*}\left(F_{t_{n}}^{X}\right)$-system $\left\{T_{s}^{X}: s \in S\right\}\left(T_{\emptyset}=F_{t_{n}}^{X}\right)$ and its extension $\delta^{*}\left(F_{t_{n}}^{X}\right)=\left\{X_{s}: s \in S\right\}\left(X_{\emptyset}=O_{t_{n}}^{X}\right)$. Using the open sets $f_{t_{n}}\left(T_{t_{n}}^{X}\right)$ we construct the $\gamma\left(F_{t\left(t_{n}\right)}^{Y}\right)$-system $\left\{T_{s}^{Y}=f_{t_{n}}\left(T_{t_{n}}^{X}\right): s \in S\right\}$ and an extension $\delta\left(F_{t\left(t_{n}\right)}^{Y}\right)=\left\{Y_{s}: s \in S\right\}$ to $Y$.

It is well known (see the proof of Theorem 7.3.15 in [2]) that for a given $\varepsilon>0$ every open cover of the open subset $Z_{s}^{X} \subset X$ has a refinement consisting of clopen (in $X$ ) pairwise disjoint sets of diameter less than $\varepsilon$. Then, by (iv) we may suppose that $Z_{s}^{X}=\bigcup \lambda_{s}^{X}$ and $Z_{s}^{Y}=\bigcup \lambda_{s}^{Y}$, where $\lambda_{s}^{X}$ and $\lambda_{s}^{Y}$ are families of clopen pairwise disjoint sets of diameter less than $1 / 2^{n+1}$ and there is a surjection $t: \lambda_{s}^{X} \rightarrow \lambda_{s}^{Y}$. Denote by $\tau_{n+1}^{X}=\left\{O_{t_{n+1}}^{X}\right\}$ and $\tau_{n+1}^{Y}=\left\{O_{t_{n+1}}^{Y}\right\}$ the families of elements of all the obtained families $\lambda_{s}^{X}$ and $\lambda_{s}^{Y}$. Choosing the sets $O(x)$ in the proof of Proposition 0 such that $[O(x)] \subset U_{n}^{X}$ we may suppose that $O_{t_{n+1}}^{X} \subset U_{n}^{X}$. Let $F_{t_{n+1}}^{X}$ be the first nonempty intersection of $O_{t_{n+1}}^{X}$ with $F_{i}$. By analogy we construct the sets $F_{t\left(t_{n+1}\right)}^{Y}$ in $Y$ and obtain open maps

$$
f_{t_{n+1}}: F_{t_{n+1}}^{X} \rightarrow F_{t\left(t_{n+1}\right)}^{Y} \text {. }
$$

Now we define $g_{\sigma}: F_{\sigma}^{X} \rightarrow F_{\sigma}^{Y}$ and $g_{\delta}: G_{\delta}^{X} \rightarrow G_{\delta}^{Y}$. By definition, $g_{\sigma}\left\lceil F_{t_{n}}^{X}=f_{t_{n}}\left(t_{0}=0\right)\right.$. It is clear that $g_{\sigma}: F_{\sigma}^{X} \rightarrow F_{\sigma}^{Y}$ is a surjective map. Note that by the construction we have

$$
g_{\sigma}\left(O_{t_{n}}^{X} \cap F_{\sigma}^{X}\right)=O_{t\left(t_{n}\right)}^{Y} \cap F_{\sigma}^{Y} .
$$

In order to define $g_{\delta}: G_{\delta}^{X} \rightarrow G_{\delta}^{Y}$ first note that by (a) and (b) for every sequence

$$
O_{t_{1}}^{X} \supset \ldots \supset O_{t_{n}}^{X}
$$

there is some

$$
x \in \bigcap_{t_{n}} O_{t_{n}}^{X}=\bigcap_{t_{n}}\left[O_{t_{n}}^{X}\right] \subset G_{\delta}^{X} .
$$

Conversely, for every $x \in G_{\delta}^{X}$ there is a sequence (2). For $x \in G_{\delta}^{X}$ defined by (2) put $g_{\delta}(x)=y=\bigcap_{t\left(t_{n}\right)} O_{t\left(t_{n}\right)}^{Y} \subset G_{\delta}^{Y}$. By (a) and (b) we 
obtain a surjection $g_{\delta}: G_{\delta}^{X} \rightarrow G_{\delta}^{Y}$, since, by our construction, for every $y \in G_{\delta}^{Y}$ there is a sequence $O_{t\left(t_{1}\right)}^{Y} \supset \ldots \supset O_{t\left(t_{n}\right)}^{Y}$ (containing $y$ ), and, hence, for the $x$ defined by (2), we have $g_{\delta}(x)=y$ and

$$
g_{\delta}\left(O_{t_{n}}^{X} \cap G_{\delta}^{X}\right)=O_{t\left(t_{n}\right)}^{Y} \cap G_{\delta}^{Y} .
$$

It remains to define $g: X \rightarrow Y$ as follows:

$$
g(x)= \begin{cases}g_{\sigma}(x) & \text { if } x \in F_{\sigma}^{X} \\ g_{\delta}(x) & \text { if } x \in G_{\delta}^{X}\end{cases}
$$

FACT. The surjection $g: X \rightarrow Y$ is an open continuous map from $X$ onto $Y$.

Indeed, by (1) and (3) we have

$$
g\left(O_{t_{n}}^{X}\right)=O_{t\left(t_{n}\right)}^{Y} .
$$

Let $x \in G_{\delta}^{X}$. Take for $x$ the sequence (2) of sets $O_{t_{n}}^{X} \ni x$. Obviously, they constitute a base at $x$, and the $O_{t\left(t_{n}\right)}^{Y}$ are a base at $g(x)$, hence $g: X \rightarrow Y$ is an open continuous map.

Let $x \in F_{\sigma}^{X}$; hence, for some $F_{t_{n}}^{X}, x \in F_{t_{n}}^{X}$. Let $\mathcal{B}=\left\{X_{s}: x \in X_{s}\right\}$ be a base at $x$, where $X_{s}$ is constructed as above. Since

$$
X_{s}=F_{t_{n}}^{X} \cup \bigcup\left\{Z_{p}^{X}: Z_{p}^{X} \subset X_{s}, p \in S\right\}
$$

and every $Z_{p}^{X}$ is the union of some $O_{t_{n+1}}^{X}$ for which

$$
g\left(O_{t_{n+1}}^{X}\right)=O_{t\left(t_{n+1}\right)}^{Y} \subset Z_{p}^{Y}
$$

we see that $g\left(X_{s}\right)=Y_{s}$, hence $g$ is an open map at $x$.

Lemma 4. Let $X, Y$ be metric spaces, $T$ be closed in $X, \operatorname{dim} X=0$ and for all nonempty open sets $U \subset X \backslash T, V \subset Y$, there exists an open map $\varphi: U \rightarrow V$. Then every open map $f: T \rightarrow Y$ can be extended to an open map $g: X \rightarrow Y$.

Proof. According to Section 2, consider a $\gamma^{*}(T)$-system and its $\delta^{*}(T)$ extension in $X$. If $Z_{s} \neq \emptyset$ then there exists an open map $\varphi_{s}: Z_{s} \rightarrow f\left(T_{s}\right)$. Let $g\left\lceil Z_{s}=\varphi_{s}\right.$ and $g\lceil T=f$.

Obviously, we thus obtain a map $g: X \rightarrow Y$ which is open at every $x \in X \backslash T$. The sets $X_{s}$ containing $x \in T$ constitute a base at $x$. By (e) and $\left(f_{1}\right)$ of Section 2 the sets $g\left(X_{s}\right)$ constitute a base at $g(x)$, and $g$ is continuous and open.

Lemma 5. Let $T^{X} \subset X \subset B(\tau)$ and $T^{Y} \subset Y \subset B(\tau)$ be closed n.d. sets in $X$ and $Y$, and $f: T^{X} \rightarrow T^{Y}$ be an open map. Suppose that for every (nonempty) open $V \subset X \backslash T^{X}$ and $U \subset Y \backslash T^{Y}$ there exists an open map $\varphi: V \rightarrow U$. Then $f$ has an open extension $g: X \rightarrow Y$ over $X$. 
Proof. The proof is, to some extent, similar to the proof of Lemma 3 or Lemma 4. (Define $g: X \rightarrow Y$ as follows: $g \nmid T^{X}=f, g\left(Z_{s}^{X}\right)=Z_{s}^{Y}$, where $g \uparrow Z_{s}^{X}$ are open maps of sets chosen as at the beginning of the proof of Lemma 3.)

\section{References}

[1] F. van Engelen and J. van Mill, Borel sets in compact spaces: some Hurewicztype theorems, Fund. Math. 124 (1984), 271-286.

[2] R. Engelking, General Topology, PWN, Warszawa, 1977.

[3] F. Hausdorff, Über innere Abbildungen, Fund. Math. 23 (1934), 279-291.

[4] W. Hurewicz, Relativ perfekte Teile von Punktmengen und Mengen (A), ibid. 12 (1928), 78-109.

[5] L. V. Keldysh, On open maps of analytic sets, Dokl. Akad. Nauk SSSR 49 (1945), 646-648 (in Russian).

[6] K. Kuratowski, Topology, Vol. I, Academic Press, 1976.

[7] S. V. Medvedev, Zero-dimensional homogeneous Borel sets, Dokl. Akad. Nauk SSSR 283 (1985), 542-545 (in Russian).

[8] J. van Mill, Characterization of some zero-dimensional separable metric spaces, Trans. Amer. Math. Soc. 264 (1981), 205-215.

[9] A. V. Ostrovsky, Concerning the Keldysh question about the structure of Borel sets, Mat. Sb. 131 (1986), 323-346 (in Russian); English transl.: Math. USSR-Sb. 59 (1988), 317-337.

[10] -, On open mappings of zero-dimensional spaces, Dokl. Akad. Nauk SSSR 228 (1976), 34-37 (in Russian); English transl.: Soviet Math. Dokl. 17 (1976), 647-654.

[11] - On nonseparable $\tau$-analytic sets and their mappings, Dokl. Akad. Nauk SSSR 226 (1976), 269-272 (in Russian); English transl.: Soviet Math. Dokl. 17 (1976), 99-102.

[12] -, Cartesian product of $F_{\mathrm{II}}{ }^{-s p a c e s}$ and analytic sets, Vestnik Moskov. Univ. Ser. Mat. 1975 (2), 29-34 (in Russian).

[13] - Continuous images of the product $\mathbb{C} \times \mathbb{Q}$ of the Cantor perfect set $\mathbb{C}$ and the rational numbers $\mathbb{Q}$, in: Seminar on General Topology, Moskov. Gos. Univ., Moscow, 1981, 78-85 (in Russian).

[14] J. Saint Raymond, La structure borélienne d'Effros est-elle standard?, Fund. Math. 100 (1978), 201-210.

MARINE TECHNICAL UNIVERSITY

LOTZMANSKAYA STR. 3

ST. PETERSBURG, 190006, RUSSIA

Received 31 March 1993;

in revised form 22 December 1993 and 14 June 1994 